\title{
On the design and realization of novel pseudo-random based chaotic signal generator for CDMA applications
}

\author{
G. Mohiuddin Bhat, Javaid A. Sheikh ${ }^{1}$ and Shabir A. Parrah ${ }^{1}$ \\ University Science Instrumentation Centre, University of Kashmir, Srinagar-190006-India, \\ ${ }^{1}$ Post Graduate Dept. of Electronics \& Instrumentation Technology, University of Kashmir, Srinagar-190006-India \\ sjavaid_29ku@yahoo.co.in; shabireltr@gmail.com
}

\begin{abstract}
In this paper, a novel Pseudo-random (PN) based wideband chaotic signal generator is proposed. The PN code generated has been used to simulate a digitally controllable variable resistor. The value of resistor and hence the frequency of chaotic signal generator is made vary by incoming PN code. The results of the implemented scheme used to generate wide range of frequencies of random nature are incorporated in the paper.
\end{abstract}

Keywords: Chaotic Signal Generator; CDMA; PN Code; GPS; radar tracking system.

Introduction

One of the major hurdles in Code Division Multiple Access Technology is the synchronization of code used at transmitting end and the one utilized at receiving end. For very long, numerous contributions of varying success have been made by researchers around the world for the synchronization of multiple access communication systems. Dynamical (Chaotic) based synchronization method is shown to be capable of providing high quality synchronization, which is essential for multiple access communication technology. The Pseudo-random (PN) Code has been widely used in spread spectrum modulation for achieving synchronization and security besides other applications (Bhat et al., 2009), but due to lack of complexity of pseudo-random codes it can not find applications in areas like multi-user communication system, Global Positioning Systems, Radar Tracking Systems.

Chaotic Systems are dynamical systems which show complex behavior. One of the defining attributes of chaotic systems is the sensitive dependence on initial conditions. Time series generated from chaotic systems are wideband in nature and noise like in appearance (Strogatz, 2001). Because of these special properties, chaotic systems are being widely studied for the multiuser secure communication technology. Efficient utilization of bandwidth is the most common technique used for electronically communicating sensitive information. However, this requires that the receiver know the "code" used by the sender to encrypt the message. In classical secure communication systems, this key, which is typically a sequence of numbers, is usually send over a secure channel while the encrypted messages is transmitted over a public channel. It would, be of course very useful, if the key need not

Research article

CIndian Society for Education and Environment (iSee) to be transmitted at all. The spread spectrum communication has been used in satellite communication for TV broadcasting, Telephony and bundled data tracking, Military communication, and data relay for space missions (Halford \& Brandit-Parce, 2000). Satellite can provide service to mobile users as well as fixed ground users while the terrestrial networks (fiber and wireless) can only cover terrestrial users on several approaches have recently been proposed for secure communications using synthesized chaotic systems. Chaotic Communications based on the transmission of messages encoded on a chaotic waveform are a subject of great current interest, attracting increasingly intensive research activities compared to conventional communication systems; there are several unique features of chaotic communication systems. Potential benefits of chaotic communications include efficient use of the bandwidth of a communication channel, utilization of the intrinsic nonlinear-ties in communication devices, large signal modulation for efficient use of carrier power, reduced number of components in a system, and security of communication by chaotic encryption. In this paper, a novel technique for the generation of chaotic signal for various communication applications has been presented.

\section{Chaotic dynamics}

Chaotic systems are nonlinear dynamic of certain distinct characteristics. These systems can generate highly complex waveforms even though the number of interacting variables is minimal. For an iterated map a dynamical system with single variable can result in

Fig. 1. Block diagram representation of proposed chaotic signal generator

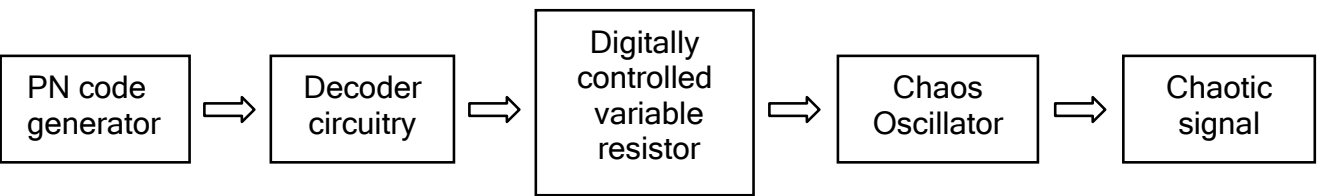




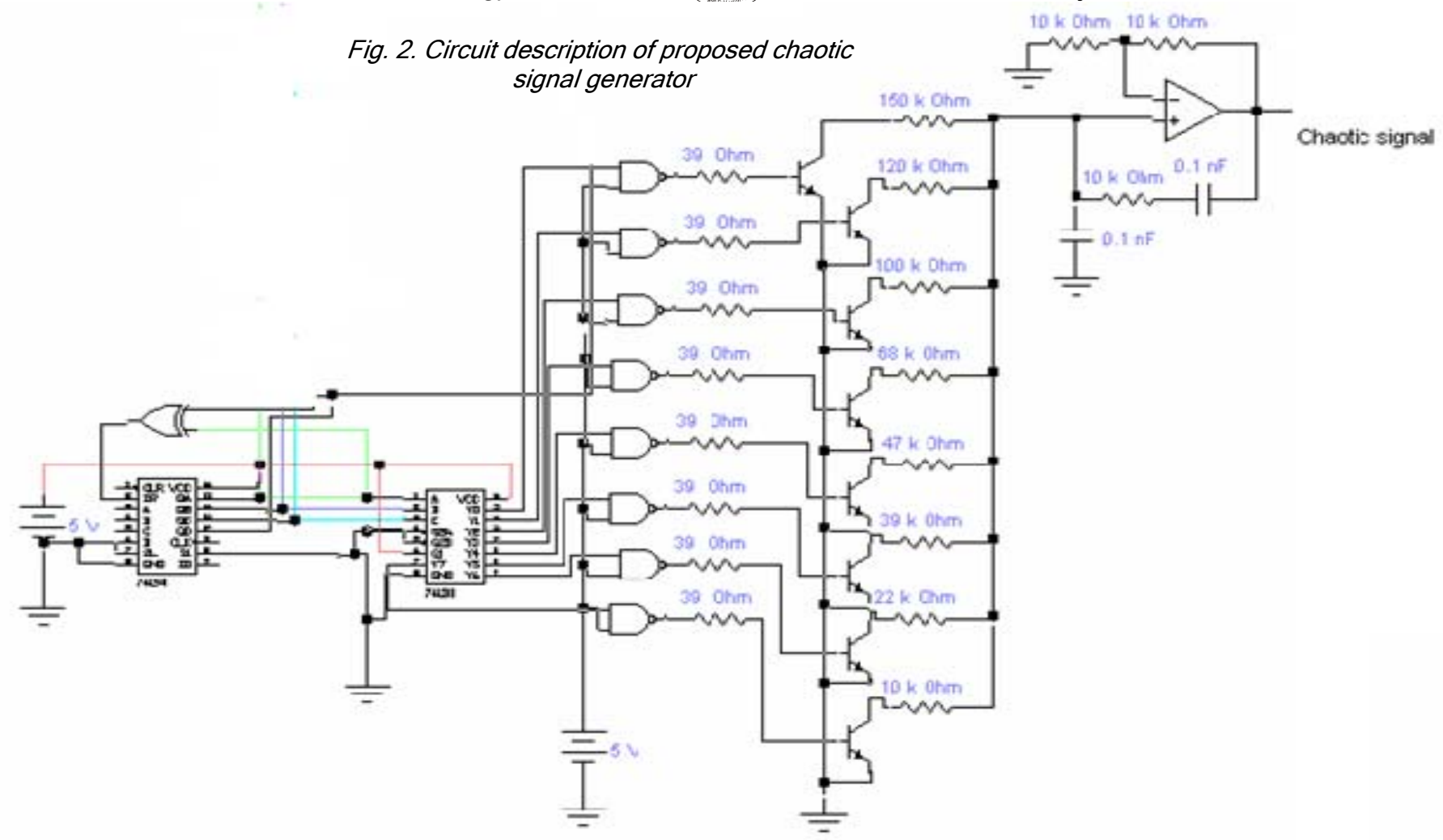

Vol. 3 No. 5 (May. 2010)

ISSN: 0974- 6846

Fig. 2. Circuit description of proposed chaotic

chaotic behavior while for a continuous system, three coupled differential equations can result in a complicated dynamics. Time series generated from chaotic dynamics have the following three interesting properties: (i) wide band spectrum, (ii) noise like appearance, and (iii) high complexity. In a chaotic system, trajectories starting from slightly different initial conditions diverge exponentially in time. This is called the sensitive dependence on the initial conditions. Because of these distinctive properties, chaotic systems are widely being studied for secure communication applications. Basically there are two ways by which chaos can be used in communication system: (i) To use chaotic time series as wide-band carrier so that coding and modulation can be accomplished together (e.g., CSK, frequency modulated differential chaotic shift keying (FM-DCSK), and (ii) To use chaotic sequences as an alternative source for spreading sequences in direct sequence spread spectrum (DS/SS) and Frequency Hopping Spread Spectrum (FH/SS) communication systems.

\section{Pseudo-Random code based chaotic signal generator}

In the proposed scheme, the Pseudo-random (PN) code generated by Linear Feedback Shift Register (LFSR) has been used to drive $3 \times 8$ decoder. The outputs of the decoder are given as one of the inputs to the array of two input AND Gates. The second input to the AND Gates are kept high. The decoder- AND combination is used to switch one of the transistors, used in transistor array, randomly. The emitters of all the transistors have been grounded, while as collectors are tied together to realize a digitally controllable resistor, that replaces the resistor of the parallel RC leg of a conventional oscillatory circuit. The output of the oscillator produces the sustained chaotic signal with varying frequency and amplitude. The proposed scheme generates wide range of frequencies from few $\mathrm{KHZ}$ to megahertz range which is suitable for data spreading in spread spectrum modulation technology. The block and circuit diagram of the proposed scheme is depicted in Fig. 1 and 2 respectively. The waveforms of different frequencies, as obtained from the implemented circuit have been presented in Fig. 3 .

\section{Experimental verification and results}

The Proposed technique for chaotic signal generator has been experimentally verified by using readily available non-linear and linear IC's. The conventional PN code generated by Linear Feedback Shift Register (LFSR) has been used for controlling the operation of decoder circuitry which in turn is used to produce output of varying frequency in a random fashion. The decoder and digitally controlled variable resistor have also been implemented using readily available IC's. The resultant chaotic signal generator generates a wide band of from of frequencies from few $\mathrm{KHZ}$ to megahertz range. The proposed technique is available as a hardware module. The waveforms obtained are satisfactory to conformity.

\section{Conclusion}

A new technique for generation of chaotic signals for multi user communication has been proposed. The proposed scheme is capable of providing a range of
Research article

CIndian Society for Education and Environment (iSee)
"Chaotic signal generator" http://www.indjst.org
Bhat et al. Indian J.Sci.Technol. 
Applications in Spread Spectrum Modulation, Code Division Multiple Access and Global Positioning System. Moreover, the proposed technique has a potential of introducing a high degree of security with low complexity. The conventional PN code has been taken as a reference signal which after processing controls the operation of digitally controlled variable resistor so as to enable the use of chaotic signal generation for Spread Spectrum Modulation

technique. Moreover, while using chaotic signal for spreading of message signal eliminates the requirement synchronization of transmitter and receiver. The chaotic signal has a potential of showing good cross-correlation, auto-correlation and other statistical properties. The experimental

investigations carried out on the hardware modules have proved the efficacy of the proposed scheme. The results have been presented in the form of various waveforms.

\section{References}

1. Bhat G.M, Mustafa M, Shabir Ahmad and Javaid Ahmad (2009) VHDL modeling and simulation of data scrambler and descrambler for secure data communication. Indian J.Sci.Technol. 2 (10), 41-43. Domain site: http://www.indjst.org.


Vol. 3 No. 5 (May. 2010)

ISSN: 0974- 6846
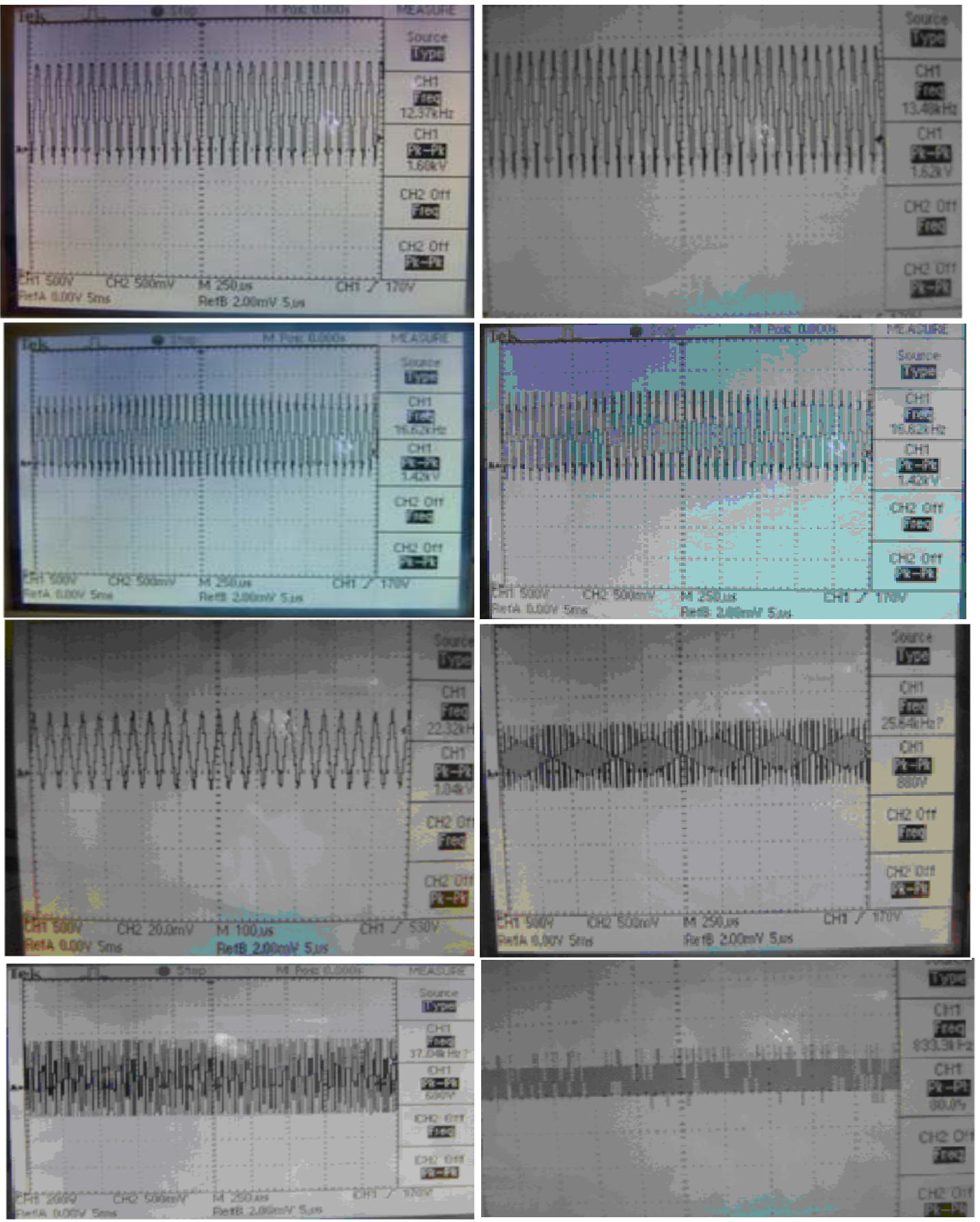

2. Halford $\mathrm{K}$ and Brandit-Parce (2000) Multistage multiuser detection for mobile FHMA. IEEE Trans. Commun. 48(9), 1550-1562.

3. Strogatz SH (2001) Nonlinear Dynamics and Chaos: with applications to Physics, Biology, Chemistry and Engineering, Persous Books, Cambridge MA. 